\title{
BMJ Open Curricula and learning objectives in nurse practitioner programmes: a scoping review protocol
}

\author{
Birgitta Ljungbeck, ${ }^{1,2}$ Katarina Sjogren Forss, ${ }^{1}$ Hafrún Finnbogadóttir, ${ }^{1}$ \\ Elisabeth Carlson ${ }^{1}$
}

To cite: Ljungbeck B, Forss KS, Finnbogadóttir $\mathrm{H}$, et al. Curricula and learning objectives in nurse practitioner programmes: a scoping review protocol. BMJ Open 2019;9:e028699. doi:10.1136/ bmjopen-2018-028699

- Prepublication history for this paper is available online. To view these files, please visit the journal online (http://dx.doi. org/10.1136/bmjopen-2018028699).

Received 19 December 2018 Revised 22 June 2019 Accepted 04 July 2019

Check for updates

(c) Author(s) (or their employer(s)) 2019. Re-use permitted under CC BY-NC. No commercial re-use. See rights and permissions. Published by BMJ.

${ }^{1}$ Department of Care Science, Faculty of Health and Society, Malmö University, SE 20506, Sweden

${ }^{2}$ Municipal Healthcare in Hässleholm, Management of Care and Welfare, Löjtnants GranlundsVäg 12, SE 28152, Sweden

Correspondence to Birgitta Ljungbeck; birgitta.ljungbeck@mau.se

\section{ABSTRACT}

Introduction Globally, nurse practitioner (NP) has become an important nursing role in the pursuit of a more efficient healthcare, possessing the necessary expert skills to work as autonomous practitioners. Nevertheless, there are barriers in the implementation of this role. One barrier concerns the different levels of education required for NPs. Previous studies demonstrate the importance of acting for a uniform international education. The aim of the scoping review was to compile research about education to become an NP, focusing on the content of curricula and learning objectives.

Methods and analysis The six-stage methodological framework by Arksey and 0'Malley will guide the scoping review through the following stages: identifying the research questions; identifying relevant studies; study selection; charting the data; collating, summarising and reporting the results; and consultation. The research questions are as follows: What is the content of curricula in NP programmes? What are the learning objectives in NP programmes? The literature searches will be conducted between March and June 2019 in the following databases: PubMed, CINAHL and ERIC, followed by hand searching reference lists of key studies. Grey literature will be searched in Google Scholar, Libsearch, existing networks and relevant organisations. Two researchers will screen titles and abstracts. Included full-text articles will be screened by three researchers and assessed for their methodological quality by the use of the Critical Appraisal Skills Programme. The PreferredReporting Items for Systematic Reviews and Meta-Analyses flow diagram will be used to demonstrate included and excluded articles. The findings will be presented through a numerical summary of the included articles, followed by a thematic analysis.

Ethics and dissemination Research ethics approval is not required for a scoping review. The scoping review will be submitted to a peer-reviewed journal. Additionally, the findings will be disseminated to stakeholders representing political, educational, professional and union organisations through a Delphi study as part of the consultation stage of the Arksey and 0'Malleys framework.

\section{INTRODUCTION}

Globally, nurse practitioner (NP) has become an important new nursing profession necessary to make healthcare more effective. ${ }^{1-3}$

\section{Strengths and limitations of this study}

- The scoping review methodology is appropriate to use when a topic is not extensively reviewed as with research on education to become a nurse practitioner.

- The search strategy is developed in collaboration with two research librarians with expertise in literature searching.

- The literature search will include both published and unpublished (grey) literature.

- No limitations will be set for the search dates.

- Only literature in English or Swedish will be included.

The growing group of frail elderly suffering from chronic diseases place extra burden on a strained healthcare and is a challenge to meet for countries worldwide. ${ }^{45}$ Studies show that NPs' entry in the healthcare system has contributed to increased patient quality (ie, a more person-centred care based on the patients' needs). In addition, NPs have the necessary skills to work as autonomous practitioners with extended responsibility previously held only by doctors. Consequently, there are more practitioners who can make medical diagnosis and treat patients, which has contributed to increased accessibility to healthcare. ${ }^{1-3}$ The International Council of Nurses (ICN) define the role of NP as a 'a registered nurse that has the required expert knowledge, the ability to make complex decisions and clinical competence for an expanded work description, whose character is formed by the context and/or the country where he/she has the right to work' ${ }^{6}$ However, there is no clear definition of this role and different titles are used, contributing to confusion when developing the professional NP role. ICN describes that advanced practice nurse (APN) is used as an umbrella term to elucidate the variety of the advanced nursing roles that currently exist. ${ }^{6}$ The most common titles included in this concept are 
NP, APN, advanced NP and clinical nurse specialist, ${ }^{1}$ but as many as 13 different titles are found in the concept of APN. ${ }^{5}$ In this scoping protocol, the title NP will be used.

ICN has a global impact on the development of NP roles globally and has provided stakeholders with information regarding role development, education, research, policy and regulatory developments. ${ }^{7}$ ICN highlights two essential components in their description of core competencies for NPs, termed as 'practice competencies' and 'professional development competencies'. These elements include not only expert skills sufficient to become autonomous practitioners but also expert skills in leadership, research and evaluation of healthcare. ${ }^{7}$ Furlong and Smith $^{8}$ refer to a similar description of core competencies for NPs divided into four attributes of expert skills. These include autonomy in clinical practice, pioneering professional and clinical leadership, expert practitioner and researcher. ${ }^{8}$ Common denominators for both descriptions of core competencies include expert skills in advanced health assessment, diagnosis, disease management, health education and promotion, referral ability, prescribing diagnostic procedures, medication and treatment plans, admitting and discharging privileges, patient caseload management, collaboration practice, evaluation of healthcare services and research. ${ }^{78}$

ICN recommends that NP programmes lead to a master's degree. ${ }^{7}$ However, in many countries, there are shortcomings regarding the frameworks that regulate these programmes, and not all are in line with ICN guidelines. ${ }^{5} 9$ Rather, some countries are educating NPs at lower academic levels, and concerns have been raised whether the curricula of these NP educational programmes provide sufficient knowledge to achieve the necessary skills. ${ }^{8-10}$ MacKay et $a l^{11}$ investigated how well prepared NPs $(\mathrm{N}=159)$ felt after completion of an NP programme, and more than $50 \%$ stated that they were not prepared for the work they were expected to handle. Further, more than $60 \%$ stated that they lacked both theoretical and practical knowledge. ${ }^{11}$ It is worth noting that this study does not reveal which level of education the participants had undergone to become an NP. However, other studies indicate that differences in education levels complicate the development of this advanced nursing role. Lack of a uniform education leads to the role of NP being perceived as a diffuse and unclear role, not readily accepted in healthcare teams. ${ }^{912}$

An international study with 31 participating countries revealed that $71 \%(\mathrm{n}=22)$ have a formal NP/APN education. Of these, 20 countries stated that the most recurrent qualifications were master's degree $(n=10)$, bachelor's degree $(n=5)$, associate degree $(n=4)$ or certificate $(\mathrm{n}=3) .{ }^{13}$ In contrast, O'Rourke et $a l^{14}$ found in their investigation of NPs $(\mathrm{N}=632)$ from 49 states in the USA that $83.7 \%(n=529)$ of the participants held a master's degree. Just over $10 \%$ had a higher level and held a doctor of nursing practice degree or a doctor of philosophy degree, and only $4.7 \%$ had a lower education. ${ }^{14}$ The reason for this is that in the English speaking countries
(USA, Australia, UK, Ireland and Canada), the role of NP is established in the healthcare systems compared with countries where the role of NP is still developing. Nevertheless, in the UK, there is great variation in the level of education. MacKay et $a l^{11}$ highlight that there is a lack of research related to NP educational programme structures and the content of these curricula. They emphasise that more research is needed to ensure that an NP degree holds the expected learning outcomes. ${ }^{11}$

From a Swedish perspective, the role of NP is slowly developing, but a legislated framework is still missing. Some minor attempts have been initiated to develop the role in collaborations between universities and local healthcare settings, but to date, the role has not achieved national attention. ${ }^{15-17}$ Nevertheless, in a recent report ${ }^{18}$ from the Swedish Government's Official Investigation, the need to develop this role is highlighted as a means of addressing future healthcare demands. In the report, it is argued that NPs will increase the quality of care for the patients, emphasising the particular value for frail elderly with complex nursing and medical needs. ${ }^{18}$ To establish the appropriate level of theoretical and practical knowledge in the development of education programmes for NPs in a Swedish context, further investigation is necessary. Therefore, the main objective of this scoping review was to systematically review the literature to identify specific features of curricula and learning objectives that are recurrent in NP education programmes globally.

\section{METHODS \\ Design}

A scoping review can be used when a topic has not yet been extensively reviewed or is of a complex or heterogeneous nature. One strength of a scoping review is that it can address a broader research question and include a variety of study designs, compared with a systematic review where the focus is on well-defined questions with a limited variety of study designs. ${ }^{19}{ }^{20}$ Another strength is that the scoping methodology allows a flexibility to search both published and unpublished (grey) literature. Additionally, a scoping review may be of value to identify research gaps in the existing literature. ${ }^{19-21}$ The present scoping review will build the six-stage methodological framework by Arksey and O'Malley, which includes identifying the research questions; identifying relevant studies; study selection, charting the data, collating, summarising and reporting the results and consultations ${ }^{19-22}$

\section{Stage 1: identifying the research questions}

Arksey and O'Malley emphasise that the research question in a scoping review should be broad, but they point out the importance of having a clearly articulated scope of inquiry. ${ }^{22}$ A population, interestPICo model (table 1) is suitable to help formulate the research questions. ${ }^{23}$ As few previous studies focus on the content of curricula and learning objectives, ${ }^{12}$ one primary research question 
Table 1 Overview PICo

\begin{tabular}{lll}
\hline $\mathbf{P}$ (population) & I (interest) & Co (context) \\
\hline Nurse practitioners & $\begin{array}{l}\text { Content of curricula and learning objectives } \\
\text { in nurse practitioner programmes }\end{array}$ & $\begin{array}{l}\text { Compilation of nurse practitioner } \\
\text { programmes internationally }\end{array}$ \\
\hline
\end{tabular}

PICo, population, interest, context.

followed by two supplementary questions will guide the scoping review.

- What is known about the educational programmes to become an NP?

- What is the content of curricula in NP programmes?

- What are the learning objectives in NP programmes?

\section{Stage 2: identifying relevant studies}

A scoping study requires inclusion and exclusion criteria. ${ }^{19}$ Studies will be included if they are written in English or Swedish, published in peer-reviewed journals, and focused on the content of curricula and learning objectives in education of NPs, and excluded if they do not correspond with these criteria. No limitations will be set for dates. Studies will be sought in the following databases: CINAHL, PubMed and ERIC. Supplementary search to find grey literature will be performed in Google Scholar, Libsearch and existing networks, relevant organisations and hand searching of reference lists in the included studies. A search strategy is created in collaboration with two research librarians with expertise in literature searching. The search strings are developed through use of subject headings (Medical Subject Headings and thesaurus) together with other free-text keywords. To expand and to narrow the search, the Boolean operators OR/AND will be used. To find relevant terms, an initial search was performed, and the terms were tested in different combinations before the definite search strategy was constructed. This resulted in four search strings: nurse practitioner OR advanced practice nurs* OR advanced practice registered nurse* $O R$ advanced nurse practitioner* OR clinical nurse specialist* AND education OR education* programme* OR education nursing graduate OR education nursing masters OR education competency based OR problem-based education OR education distance OR course* AND curriculum OR curricul* OR content of curricul* OR curricul* evaluation* OR syllabi OR syllabus OR course syllabi OR course syllabus AND learning objectives OR learning outcome* OR education* preparation OR education* objectives* OR education outcomes. A summary of the search will be presented in a table. The literature search will be conducted between March 2019 and June 2019. The following screening and analysis of the literature will be completed no later than September 2019.

\section{Stage 3: study selection}

The study selection will be performed in several steps. Levac et $a l^{19}$ emphasise that the study selection is a critical step that requires at least two researchers who the abstracts independently of each other read . Primarily, a screening of titles will take place by one researcher followed by a more comprehensive reading of abstracts by two researchers. When appropriate abstracts are identified, the articles will be read in full text and compared with the inclusion and exclusion criteria. The potential studies will be assessed for their methodological quality by use of the Critical Appraisal Skills Programme consisting of a number of crucial criteria that should be included in the studies to determine whether the studies are of good quality or not. ${ }^{24}$ If agreement is not reached between the two researchers who are involved in the study selection, a third research member will be consulted. ${ }^{19}$ The web-based tool Mendeley will be used for organising articles and removing duplicates, as well as the PreferredReporting Items for Systematic Reviews and Meta-Analyses (PRISMA) flow diagram for illustrating the process of inclusion and exclusion of articles. ${ }^{25}$

\section{Stage 4: charting the data}

Three researchers will read the included full-text articles independently of each other, extract data that respond to the research questions and organise the data in key themes. To organise and manage the extracted data, we will use a charting form including a description of why each study is included or excluded. If there is any discrepancy between the researchers, a fourth researcher will be consulted until consensus is reached. ${ }^{19}{ }^{20}$ Levac et $a l^{19}$ emphasise the importance of involving the research team to determine appropriate variables and to start the extraction of the data. This step should be seen as an iterative process, and it is necessary to review the included articles several times to be sure that the chosen data extraction corresponds to the research question and the aim of the study. ${ }^{19}$ To increase the reliability of the findings, demonstrate methodological accuracy and enable replications by others, each step in the process will be documented using the checklist PRISMA Extension for Scoping Reviews (Prisma-ScR). ${ }^{25}$

\section{Stage 5: collating, summarising and reporting the results}

According to Levac et $a l,{ }^{19}$ this step includes analysing the data, reporting results and applying meaning to the results. We will do this by presenting a numerical summary of the included articles, followed by a thematic analysis. Study characteristics to be extracted will include, but not be limited to publication year, publication type (ie, original research), study design, country, description of curricula and learning objectives, and level of NP programmes. The thematic analysis aims to structure 
and organise the extracted data in themes that reflect the outcomes. Finally, the result will be presented and summarised based on these themes. ${ }^{19}$ To handle the data in the thematic analysis, the data analysis programme NVivo V.11 will be used to support the discovery of patterns, similarities and differences. ${ }^{26}$

\section{Stage 6: consultation}

This final step is not always used because it adds a time-consuming supplementary process of analysing and integrating new data with the previous results. ${ }^{22}$ However, findings from this scoping review will subsequently be linked to a Delphi study focusing educational issues to become an NP. Participants from political, educational, professional and union organisations will be invited. Consensus methods such as the Delphi survey technique are being employed to help enhance effective decision-making in health and social care. The Delphi survey is a group facilitation technique, which is an iterative multistage process, designed to transform opinions into group consensus. ${ }^{27-29}$

Contributors BL drafted the study protocol and led the construction of the search strategy. The coauthors, KSF, HF and EC, were involved by giving continuous feedback regarding the methodological issues and the design of the manuscript. All authors gave their approval to publishing this study protocol.

Funding This research received no specific grant from any funding agency in the public, commercial or not-for-profit sectors.

Competing interests None.

Patient consent for publication Not required.

Provenance and peer review Not commissioned; externally peer reviewed.

Data availability statement All data relevant to the study are included in the article or uploaded as supplementary information.

Open access This is an open access article distributed in accordance with the Creative Commons Attribution Non Commercial (CC BY-NC 4.0) license, which permits others to distribute, remix, adapt, build upon this work non-commercially, and license their derivative works on different terms, provided the original work is properly cited, appropriate credit is given, any changes made indicated, and the use is non-commercial. See: http://creativecommons.org/licenses/by-nc/4.0/.

\section{REFERENCES}

1. Goldberg S, Cooper J, Russell C. Developing advanced nursing skills for frail older people. Nurs Older People 2014;26:20-3.

2. Donald F, Martin-Misener R, Carter N, et al. A systematic review of the effectiveness of advanced practice nurses in long-term care. $J$ Adv Nurs 2013;69:2148-61.

3. Kutzleb J, Rigolosi R, Fruhschien A, et al. Nurse practitioner care model: meeting the health care challenges with a collaborative team. Nursing Economics 2015;6:297-305.

4. Yach D, Hawkes C, Linn-Gould C, et al. The global burden of chronic diseases: overcoming impediments to prevention and control. JAMA 2004;21:2616-22.

5. Delamaire M, Lafortune G. Nurses in Advanced Roles: A description and evaluation of experiences in 12 developed countries. OECD Health Working Papers, No.54, OECD Publishing, Paris, 2010. Available: http://www.oecd-ilibrary.org/social-issues-migration- health/nurses-in-advanced-roles_5kmbrcfms5g7-en Accessed 18020/ [Accessed 12 Nov 2018].

6. International Council of nurses. Available: http://icn-apnetwork.org/ [Accessed 12 Nov 2018].

7. International Council of nurses. scope of practice and standards, 2005. Available: http://international.aanp.org [Accessed 12 Nov 2018].

8. Furlong E, Smith R. Advanced nursing practice: policy, education and role development. J Clin Nurs 2005;14:1059-66.

9. Heale R, Rieck Buckley C, Buckley C. An international perspective of advanced practice nursing regulation. Int Nurs Rev 2015;62:421-9.

10. Mundiger M, Sheets Cook S, Lenz E, et al. Assuring quality and access in advanced practice nursing: a challenge to nurse educators. Journal of Professional Nursing 2000;6:322-9.

11. MacKay M, Glynn D, McVey C, et al. Nurse practitioner residency programs and transition to practice. Nurs Forum 2018;53:156-60.

12. Bryant-Lukosius D, Dicenso A, Alba D. A framework for the introduction and evaluation of advanced practice nursing roles. $J$ Adv Nurs 2004;48:530-40.

13. Pulcini J, Jelic M, Gul R, et al. An international survey on advanced practice nursing education, practice, and regulation. Journal of Nursing Scolarship 2010;42:31-9.

14. O'Rourke N, Crawford S, Morris S, et al. Political efficacy and participation of nurse practitioners. Policy, Politics \& Nursing Practice 2017:3:135-48.

15. Altersved E, Zetterlund L, Lindblad $U$, et al. Advanced practice nurses: a new resource for Swedish primary health-care teams. Int $J$ Nurs Pract 2011:17:174-80.

16. Bergman K, Perhed U, Eriksson I, et al. Patients' satisfaction with the care offered by advanced practice nurses: a new role in Swedish primary care. Int J Nurs Pract 2013;19:326-33.

17. Jangland E, Becker D, Börjeson S, et al. The development of a Swedish nurse practitioner program - a Request from clinicians and a process supported by US experience. Journal of Nursing Education and Practice 2014;4.

18. Swedish Governments Official Investigation. State Public Reports: Future Specialist Nurses - new role, new challenges. (Statens offentliga utredningar. SOU 2018:77. Framtidens specialistsjuksköterska - ny roll, nya möjligheter). Available: https:// www.regeringen.se/4ab6f6/contentassets/6f67c0e786f0491899a9 8579a002508c/framtidens-specialistsjukskoterska-ny-roll-nyamojligheter-sou-201877 [Accessed 1 Dec 2018].

19. Levac D, Colquhoun H, O'Brien KK. Scoping studies: advancing the methodology. Implementation Science 2010;5.

20. Daudt HML, van Mossel C, Scott SJ. Enhancing the scoping study methodology: a large, inter-professional team's experience with Arksey and O'Malley's framework. BMC Med Res Methodol 2013;13:1-9.

21. O'Brien $\mathrm{K}$, Colquhoun $\mathrm{H}$, Levac $\mathrm{D}$, et al. Advancing scoping study methodology: a web-based survey and consultation of perceptions on terminology, definition and methodological steps. BMC Health Services Research 2016;305:1-12.

22. Arksey H, O'Malley L. Scoping studies: towards a methodological framework. Int J Soc Res Methodol 2007;1:19-32.

23. Joanna Briggs institute. The Joanna Briggs institute Reviewers' Manual 2015. Methodology for JBI Scoping reviews. South Australia: The University of Adelaide, 2015.

24. Critical appraisal skills programme. CASP checklist, 2018. Available: https://casp-uk.net/casp-tools-checklists/ [Accessed 25 Nov 2018].

25. Tricco AC, Lillie E, Zarin W, et al. PRISMA extension for scoping reviews (PRISMA-ScR): checklist and explanation. Ann Intern Med 2018;169:467-73.

26. Silver C, Lewins A. Using software in qualitative research, a step-bystep guide. London: Sage Publications, 2007.

27. Keeney S, Hasson F, McKenna H. Consulting the oracle: ten lessons from using the Delphi technique in nursing research. J Adv Nurs 2006;53:205-12.

28. Wilkes L. Using the Delphi technique in nursing research. Nurs Stand 2015;29:43-9.

29. Hasson F, Keeney S, McKenna H. Research guidelines for the Delphi survey technique. J Adv Nurs 2000;32:1008-15. 